\title{
Laurence Théry, Le travail intenable. Résister collectivement à l'intensification du travail
}

Jean Bastien

\section{(2) OpenEdition}

1 Journals

Édition électronique

URL : http://journals.openedition.org/travailemploi/5373

DOI : 10.4000/travailemploi.5373

ISSN : 1775-416X

Éditeur

DARES - Ministère du Travail

\section{Édition imprimée}

Date de publication : 15 octobre 2006

Pagination : 72-74

ISSN : 0224-4365

\section{Référence électronique}

Jean Bastien, «Laurence Théry, Le travail intenable. Résister collectivement à l'intensification du travail », Travail et Emploi [En ligne], 108 | octobre-décembre 2006, mis en ligne le 20 décembre 2012, consulté le 05 mai 2019. URL : http://journals.openedition.org/travailemploi/5373 ; DOI : 10.4000/

travailemploi.5373

Ce document a été généré automatiquement le 5 mai 2019.

(C) La documentation française 


\title{
Laurence Théry, Le travail intenable. Résister collectivement à l'intensification du travail
}

\author{
Jean Bastien
}

\section{RÉFÉRENCE}

Laurence Théry, Le travail intenable. Résister collectivement à l'intensification du travail, La Découverte, Paris, 2006

L'intensification du travail prend des formes diverses selon les secteurs et selon les situations ; elle n'en constitue pas moins une menace pour la santé de millions de travailleurs. En beaucoup d'endroits, les contraintes de productivité se cumulent en effet avec les contraintes exercées par les clients, auxquelles s'ajoutent désormais les contraintes psychosociales, liées aux modes de gestion et déménagement actuels, avec comme conséquence une forte dégradation des situations de travail. Dans ce contexte, et dans la foulée des enquêtes menées dans le cadre du chantier Le Travail en questions, la CFDT a décidé de faire de la santé au travail un axe de revendications nouvelles. Et elle a alors, parmi d'autres actions, entrepris de former des militants dans ce domaine. La méthode - originale - utilisée a consisté dans une formation à la recherche-action à laquelle ont participé une soixantaine d'entre eux, issus d'une vingtaine de sections syndicales d'entreprises ou d'administrations, qui ont été formés sur dix-huit mois en 2004-2005, encadrés par des formateurs syndicaux, des ergonomes, des médecins du travail et des sociologues. Elle a conduit les militants concernés à choisir un secteur de leur entreprise sur lequel mener l'enquête en allant interroge les salariés, pour partager ensuite avec eux les résultats de leurs analyses. Au passage, ils ont ainsi expérimenté une posture relativement nouvelle pour de représentants du personnel, privilégiant une élaboration collective du diagnostic et des revendications, elle-même basée sur une 
analyse de détail du travail, qui visait à prendre en compte la variabilité des conditions et le vécu des salariés. Ce livre rend compte de cette expérience originale de formation à travers la recherche-action.

\section{Des formes d'intensification du travail très diverses}

2 La première partie, rédigée par François Daniellou ${ }^{1}$ donne à voir, à partir des situations analysées par les militants, les formes diverses que prend l'intensification du travail. Elle convoque ainsi successivement : la lingère qui assure, outre le repassage, la veille de nuit dans un foyer logement pour personnes âgées, le monteur sur chaîne automobile, soumis à des gestes répétitifs et au respect des cadences, l'ouvrière qui replace les biscuits sur une chaîne de conditionnement, le soudeur de pots d'échappement aux prises avec les méthodes d'organisation de production japonaises, la téléopératrice d'un centre d'appel qui n'en peut plus d'essayer de se conformer à tout ce qu'on lui demande, le conseiller de clientèle d'une compagnie d'assurance, spectateur malgré lui de la division du travail qui dégrade le service au client, la conductrice de ligne d'embouteillage, contrainte de jongler entre les trois lignes qu'elle est censée piloter en même temps, l'ouvrier de l'usine d'accessoires automobile qui fait le travail que l'automate n'arrive pas à faire, les fraiseurs de moules pour la verrerie qui ont dû renoncer à anticiper les bris d'outils et la non-qualité, les aides soignantes dans un service hospitalier de long séjour, confrontées à la situation des personnes dépendantes, les techniciennes d'intervention sociale et familiale qui peinent sur les rapports qu'on leur demande, l'employée de la société de nettoyage, gênée par les occupants et sanctionnée à cause de cela, les ouvriers sur une chaîne de découpe de volailles qui doivent s'accommoder du manque de place, et, pour finir, les ouvrières d'une exploitation viticole, auxquelles échoit le travail le moins qualifié.

3 C'est également l'occasion pour l'auteur de se pencher sur la situation faite aux femmes au travail (Laurence Théry ${ }^{2} \mathrm{y}$ reviendra dans la seconde partie), qui, bien souvent, sous le poids des stéréotypes, concentre les causes d'intensification : le faible soutien du collectif, voire l'isolement au travail, la moindre autonomie, les contraintes et les pressions extérieures, le travail répétitif, dont les effets sur la santé sont particulièrement marqués. La situation des cadres n'est pas toujours meilleure, comme le note François Daniellou, pris entre le flux d'informations descendantes qu'ils doivent traduire et faire passer auprès de leurs subordonnés et le flux remontant du terrain et de la réalité quotidienne.

Dans tous les cas, "la flexibilité et la souplesse sont les maîtres-mots ", que doivent permettre d'atteindre des réorganisations qui sont presque toujours « top-down », alors que la connaissance de la réalité du travail et la mobilisation de l'intelligence des salariés sont le plus souvent minimales. Dans ces conditions, faire ce qu'on leur demande de faire, sans leur mettre à disposition les moyens nécessaires et sans l'organisation adéquate, ne peut que constituer pour les salariés une source de frustration infinie, aux effets dommageables sur leur santé.

\section{Les pistes de transformation passent par la mise en discussion et l'implication des salariés}

5 La deuxième partie consiste dans une série de contributions de formateurs et de chercheurs qui présentent des éléments de connaissance ou de réflexion. Laurence Théry 
revient sur la démarche d'enquête approfondie et de restitution-discussion avec les salariés. La référence principale ici mobilisée est l'ergonomie, dans la mesure où c'est la mise en évidence de la variabilité des conditions du travail qui doit ouvrir des pistes de transformation. Mais la prise de conscience et la formulation collectives des problèmes sont tout aussi essentielles, dans la mesure où elles permettent de recréer du collectif et de donner de la voix à ces analyses, dans un domaine - l'organisation du travail - que les directions ont tendance à considérer comme leur chasse gardée.

Bernard Dugué ${ }^{3}$ se penche quant à lui, en sociologue et ergonome, sur les déterminants de l'intensification du travail. Celle-ci résulte le plus souvent, explique-t-il, d'une combinaison de décisions, faiblement coordonnées entre elles, généralement prises de surcroît sous l'influence d'une vision très péjorative de l'homme au travail, qui privilégie de fait des choix qui excluent tant la subjectivité des salariés que la réalité du travail, cela jusqu'aux systèmes de contrôle mis en place. Ce qui .finit par démotiver complètement les salariés. À côté de cela, les changements sont systématiquement survalorisés par les directions ; ils sont souvent mis en œuvre dans la précipitation et sans débat véritable concernant les choix effectués. "Ces évolutions, ces modes de fonctionnement et ces façons de conduire le changement doivent faire l'objet d'un regard critique. Il en va de la santé des entreprises comme de celle des salariés » (p. 113), explique l'auteur. Ce qui repose principalement sur la capacité des acteurs sociaux de l'entreprise 1) à interroger les projets pour chercher à favoriser la prise en compte des différentes logiques et la participation des salariés concernés et 2) à repérer et anticiper les effets des petites décisions, ce qui suppose qu'ils réussissent à dégager le temps nécessaire, mais aussi à retisser des liens avec les salariés (ce qui renvoie alors au travail de représentant du personnel, auquel l'auteur a consacré une thèse et un livre qui est paru l'an dernier chez Octarès).$^{4}$

7 Corinne Gaudart ${ }^{5}$ interroge quant à elle les évolutions du travail et leurs conséquences sur les travailleurs suivant leur âge et leur expérience (celles-ci prennent en effet une importance particulière dans la période de transition démographique et d'évolution technologique rapide que l'on connaît actuellement). L'empilement des contraintes se traduit par une pression temporelle croissante, or on sait que celle-ci est moins bien tolérée quand l'âge s'élève car elle réduit la possibilité pour les opérateurs de construire des compromis entre les exigences de la tâche et leurs capacités. Les coupures entre générations, liées notamment aux difficultés d'emploi, ont compromis les aides réciproques qui pouvaient exister entre classes d'âge. « Mais en nous désignant les dégâts qu'elle engendre, l'intensification nous montre aussi les moyens de lutter. Recréer des espaces de transmission dans l'entreprise permettrait de mettre du liant entre les âges et ainsi de débattre du sens du travail » (p. 137), conclut l'auteur.

La contribution de Philippe Davezies ${ }^{6}$ creuse plus profond (on pourra également consulter les documents accessibles sur son site web). Comprendre le travail, explique-t-il, implique d'accorder un intérêt à l'activité, à la façon dont s'y construit le rapport aux objets et finalement à l'investissement subjectif de celui qui le réalise. Pour le faire bien, il faut sentir le travail, et donc payer de sa personne. Configurer des ressources, que l'on tire de soi (c'est ce qui confère à mon activité son style propre, mais également constitue fondamentalement ma capacité d'agir). Ce qui en retour contribue à former mon identité. C'est tout cela qu'un changement d'organisation peut alors mettre à mal, au risque de mettre en péril ma santé, comme on le comprend sans difficulté, quand celui-ci « tranche les liens sensibles qui, d'un côté, donnent chair à l'organisation du travail et, de l'autre, 
alimentent la vie psychique du sujet» (p. 148). Mais l'auteur va plus loin en expliquant qu'au-delà des objets sur lesquels j'investis, c'est l'activité d'autrui que je vise à travers mon travail, ou plus exactement mon articulation avec elle (même si cela n'exclut pas les conflits liés aux différences de perspectives). C'est ce qui explique du reste que le collectif ait alors une double fonction au regard du développement de l'activité, de défense de l'autonomie du métier contre les pressions qui tendent à le rabattre sur les seules dimensions instrumentales, et une fonction de transmission aux individus de l'expérience accumulée pour leur éviter les erreurs et leur permettre de progresser. On en tire également l'idée que pour transformer l'organisation, il faut procurer aux acteurs la possibilité de jouer un rôle actif dans la transformation. En attendant, "Ramener ainsi dans le débat social les conflits que les salariés portent comme des questions personnelles constitue un premier niveau de protection contre le sentiment de dévalorisation et contre les pathologies du stress » (p. 165), souligne l'auteur, avant celui qui consisterait à transformer les situations.

\section{Interroger les pratiques syndicales?}

9 La troisième partie vise à faire le bilan de cette formation par la recherche-action, plutôt que des actions proprement dites menées par les équipes. On regrettera toutefois que les améliorations obtenues ne soient pas décrites, mais il était sans doute difficile d'en rendre compte, sauf à entrer dans un grand détail. Les deux premiers chapitres sont rédigés par de jeunes sociologues. Dans le premier, Cécile Guillaume ${ }^{7}$ dresse les profils des militants et les conditions de leur engagement (la plupart sont élus de CHSCT), avant de se centrer sur les bénéfices individuels qu'ils ont pu en retirer. Une première catégorie de militants, eux-mêmes exposés à des situations de travail très difficiles, a vécu la formation, tout au moins la première étape, comme une sorte de thérapie individuelle, explique-t-elle, qui leur a permis de reconsidérer ce qu'ils vivaient sous l'éclairage d'une grille d'interprétation plus large. Malgré une certaine amélioration de leurs conditions de travail, ils « semblent aujourd'hui partagés entre un accablement devant l'ampleur du process d'intensification en marche - et le sentiment qu'il faut sans cesse recommencer le travail sur le plan syndical - et une prise de conscience de la nécessité de porter le débat à un niveau plus politique » (p. 183). Une seconde catégorie de militants, plus nombreux, moins directement concernés par l'intensification du travail, a mis à profit la formation pour renouer avec l'écoute des salariés et leur ressenti subjectif. Pour tous, l'expérience a permis la construction d'un sentiment d'appartenance plus fort à l'organisation. La plupart en ont profité pour se forger des compétences nouvelles en matière de santé au travail, et certains ont renforcé ainsi leur légitimité syndicale.

Le second chapitre, rédigé par Nadine Olivier ${ }^{8}$ explore les fonctionnements collectifs. Pour la plupart des équipes, la démarche d'enquête n'a, au départ, pas été évidente, et il a souvent été difficile pour les militants de trouver la bonne distance. La rapidité d'évolution des structures d'entreprise a parfois compliqué les choses. En interne, l'équipe n'a pas toujours bénéficié du soutien du reste de la section syndicale (la répartition des rôles entre CHSCT et CE n'a pas facilité les choses). Mais les sections en question n'étaient pas non plus toutes dans la même situation en termes de poids ou d'influence dans l'entreprise. Finalement, les salariés ont été diversement associés à la restitution, même si certaines équipes ont réussi à impliquer les salariés tout au long du processus. 
11 Le chapitre suivant, rédigé par Francis Bourdon et Danielle Mezzarobba, ergonomes et formateurs syndicaux, dresse enfin un bilan de l'expérience en tant que formation syndicale, soulignant tout son intérêt notamment pour permettre à des représentants du personnel de mieux saisir leur rôle et leur permettre de dépasser une vision a minima du travail syndical. Sur un sujet aussi complexe que la santé au travail, la démarche de recherche-action permet aux militants de comprendre tout l'intérêt d'alterner phases d'observation, de questionnement et de restitution pour une analyse objective de la situation et d'impliquer les salariés à la fois dans le diagnostic et dans l'action collective destinée à remédier aux problèmes rencontrés. Ce qui appelle alors un travail collectif des militants et qui soit poursuivi sur la durée.

La conclusion revient à Laurence Théry, qui pointe notamment, après plusieurs auteurs, comme Marie-Anne Dujarier ${ }^{9}$ ou d'autres regroupés autour de Yves Clot et Dominique L'Huillier $^{10}$, que « nous assistons aujourd'hui à une crise de l'organisation; et parce qu'on ne sait pas organiser, et parce que l'on fait de l'intelligence des salariés, les « organisateurs » reportent sur ces mêmes salariés la responsabilité de la réactivité, de la rapidité et le charge de la qualité. Certaines entreprises, certains services ou ateliers totalement désorganisés ne sont rentables que parce que les salariés sont mobilisés à 200 $\%$ » (pp. 227- 228), avec le risque d'atteintes graves à la santé que cela suppose. « Il est bon de le rappeler : le travail, ce n'est pas seulement l'aliénation. Il est bon d'être fier de son travail et, à l'inverse, il est douloureux de ne pas pouvoir le faire correctement. Le travail mobilise une bonne partie de notre vie, il engage notre identité et nous permet de nous réaliser : faire du bon travail, c'est d'abord trouver un espace où ce que l'on est va s'exprimer. C'est pouvoir renvoyer au collectif une expérience singulière qui n'est pas la simple répétition du travail des autres, mais une façon d'imprimer sa patte [...]» (p. 228). A contrario, "Quand on coince les gens dans des situations où tout est décidé sans eux, où tout est décidé au mépris de leur travail, alors apparaissent les atteintes à la santé. Nous en sommes là $[. .] ».($ p. 228).

On aura compris qu'il s'agit d'un livre engagé sur un sujet probablement appelé à connaître des développements importants au cours des prochaines années.

\section{NOTES}

1. Professeur d'ergonomie à l'université de Bordeaux, directeur du Laboratoire d'ergonomie des systèmes complexes.

2. Inspectrice du travail, chargée de la santé au travail à la CFDT.

3. Docteur en sociologie et ergonome européen, chargé de recherches au Laboratoire d'ergonomie des systèmes complexes.

4. Le travail de négociation - Regards sur la négociation collective d'entreprise, Octarès, 2005.

5. Ergonome et chercheuses au CREAPT / CNRS.

6. Enseignant et chercheur en médecine et santé au travail à l'université de Lyon 1.

7. Sociologue, maitre de conférences à l'université de Lille 1. 
8. Sociologue, doctorante au Laboratoire interdisciplinaire pour la sociologie économique (CNRS/ CNAM).

9. Marie-Anne Dujarier, L'idéal au travail, PUF, 2006 ; " La division sociale du travail d'organisation dans les services ", Nouvelle revue de psychosociologie, no 1, 2006/1.

10. Yves Clot, Dominique L'Huillier, "Perspectives en clinique du travail », Nouvelle revue de psychosociologie, no $1,2006 / 1$

\section{AUTEURS}

\section{JEAN BASTIEN}

Syndex, Cabinet d'expertise comptable spécialisé dans l'assistance aux entreprises 